\title{
Deep ST
}

\section{Thomas M. Ferguson ${ }^{1}$ • Elisángela Ramírez-Cámara ${ }^{2}$ (I)}

Received: 30 June 2020 / Accepted: 2 August 2021 / Published online: 20 November 2021

(C) The Author(s) 2021

\begin{abstract}
Many analyses of notion of metainferences in the non-transitive logic ST have tackled the question of whether ST can be identified with classical logic. In this paper, we argue that the primary analyses are overly restrictive of the notion of metainference. We offer a more elegant and tractable semantics for the strict-tolerant hierarchy based on the three-valued function for the LP material conditional. This semantics can be shown to easily handle the introduction of mixed inferences, i.e., inferences involving objects belonging to more than one (meta)inferential level and solves several other limitations of the ST hierarchies introduced by Barrio, Pailos, and Szmuc.
\end{abstract}

Keywords ST $\cdot$ LP $\cdot$ Non-transitive $\cdot$ Substructural logics $\cdot$ Classical logic Metainferences $\cdot$ Semantics

\section{Introduction}

Since its introduction in a series of papers by Pablo Cobreros, Paul Egré, David Ripley and Robert van Rooij [6, 7, 16, 17], the strict-tolerant approach to paradox has been the subject of philosophical discussion sparked by the authors' suggestion that ST might not be all that different from classical logic.

On the one hand, two papers by Eduardo Barrio, Lucas Rosenblatt and Diego Tajer, and Barrio, Federico Pailos and Damian Szmuc, respectively, put forward some

The results in this paper have largely been presented in talks given to the VII Workshop on Philosophical Logic in 2018, the ASL Logic Colloquium in 2019, and the CUNY/NYU Workshop on Metainferences in 2019. The authors acknowledge the many helpful comments that were received by attendees of these talks.

Thomas M. Ferguson

tferguson@gradcenter.cuny.edu

Elisángela Ramírez-Cámara

eliramirezc@gmail.com

1 ILLC, University of Amsterdam and Arché Research Centre, University of St. Andrews, St. Andrews, Scotland

2 National Autonomous University of Mexico, Mexico City, Mexico 
interesting results: First, Barrio, Rosenblatt and Tajer discuss ST in relation to both classical logic and LP [14]. They refuse to identify ST with either logic, mainly because unlike them, ST presents a mismatch between its notions of internal and external validity. Then, in [3], Barrio, Pailos and Szmuc generalize the idea behind

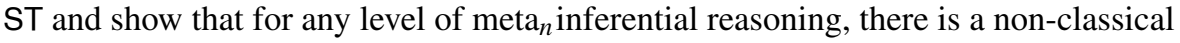
logic which takes meta ${ }_{n-1}$ inferences as its objects, and which behaves classically at all the lower levels. This leads them to propose a new criterion for the identity of logics, namely that there must be no level of inference at which the behavior of the two logics does not match. We will, in what follows, refer to this collection of results as the Buenos Aires plan (for the ST hierarchy).

On the other hand, Bogdan Dicher and Francesco Paoli have recently argued contra the Buenos Aires plan, that ST is indeed LP. Using the notion of a logic as an abstract consequence relation, they show that the behavior of the consequence relations involved in e.g. ST and LP is independent of their respective objects, and exactly the same in both cases. Consequently, they also provide strong grounds to reject the claim that ST is pretty much a different presentation for classical logic.

In this paper, we will focus on the ideas developed in [3]. We will claim that there are at least two major shortcomings in the approach to the ST hierarchy presented in that paper: it makes no room for the inclusion of mixed inferences as objects of reasoning that can be studied formally, and it seems to suggest that there might not be a clear limit to the hierarchy. Our proposal will be that these shortcomings can be remedied by providing a semantics for the hierarchy including each stage. The semantics for this limit witnesses that there is no level of metainferential reasoning that cannot be encoded by the conditional of LP. Besides providing a much needed limit, this also vastly simplifies the semantics for the hierarchy, and allows for the evaluation of mixed inferences in a rather natural way. We then close off our proposal by discussing the case of the deep ST-theorist, an individual who upholds the ST standard at all levels of reasoning. We briefly discuss some issues related to such a position and lay the groundwork for further work on the subject.

The plan of the paper is as follows. Sections 2 and 3 introduce the background needed to understand both discussions. In Section 2, we introduce the relevant formal definitions, as well as a brief overview of the strict-tolerant approach to paradox. Section 3 provides an abridged version of the results put forward by Barrio and his collaborators.

Then, Sections 4 and 5 constitute our negative and positive proposals, respectively. In Section 4, we make a case for the idea that mixed inferences are natural objects of reasoning that cannot be easily dealt with within the Buenos Aires approach to the ST hierarchy. We also note that a secondary issue is that the ST hierarchy seems to lack a clear-cut limit and we briefly describe the expectations one ought to have regarding such a limit. Section 5 proposes an alternative approach to the ST hierarchy where the main motivation is to provide a limit to the hierarchy by showing that the interaction between strict and tolerant assertion is already encoded in the LP conditional.

Before offering some concluding remarks in Section 7, we cover the case of the deep ST-theorist in Section 6. The deep ST-theorist believes that the strict-tolerant standard should be the standard of evaluation for all levels of reasoning, including those involving mixed inferences. This gives rise to some interesting issues, such as 
the deep ST-theorist's constancy with respect to inferential principles, a constancy perhaps only conspicuously seen in the case of intuitionistic reasoning; the treatment of paradox outside the object language, and the issue of whether full classicality can actually be recovered or not.

\section{Strict-Tolerant Logic}

In this section, we will make a closer survey of the primary results and intuitions underlying the strict-tolerant account of logical consequence.

\subsection{Formal Definitions}

Importantly, one of the goals behind the introduction of ST is the resolution of paradoxes of self-reference. Hence, the first-order language in which we work includes a constant term $\ulcorner\varphi\urcorner$ for every formula $\varphi$ and a truth predicate $T$ that takes such constant terms as its arguments.

For purposes of clarity, we will be agnostic with respect to the particular signature $\sigma$ of the language to be defined, assuming only that there exists a set $\mathbf{A t}_{\sigma}$ that includes atomic formulae of that signature constructed in the usual fashion.

Definition $1 \mathcal{L}_{\text {base }}$ is a first-order language in a signature with $\psi \in \mathbf{A t}_{\sigma}$ and $x \in$ Var: $\varphi::=\psi|T(\ulcorner\varphi\urcorner)| \sim \varphi|\varphi \vee \varphi| \varphi \wedge \varphi|\varphi \supset \varphi| \exists x \varphi \mid \forall x \varphi$

We now define models for such a language, pausing to make two observations. First concerns the underlying domains. The denotation of a distinguished name $\ulcorner\varphi\urcorner$ is most naturally construed as the formula $\varphi$ itself; it is thus natural to expect that the domain on which a model is built includes the language.

Second, models will evaluate complex formulae by the strong Kleene truth tables:

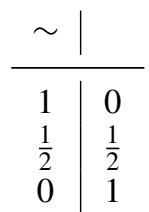

\begin{tabular}{c|ccc}
$\wedge$ & 1 & $\frac{1}{2}$ & 0 \\
\hline 1 & 1 & $\frac{1}{2}$ & 0 \\
$\frac{1}{2}$ & $\frac{1}{2}$ & $\frac{1}{2}$ & 0 \\
0 & 0 & 0 & 0
\end{tabular}

\begin{tabular}{c|ccc}
$\vee$ & 1 & $\frac{1}{2}$ & 0 \\
\hline 1 & 1 & 1 & 1 \\
$\frac{1}{2}$ & 1 & $\frac{1}{2}$ & $\frac{1}{2}$ \\
0 & 1 & $\frac{1}{2}$ & 0
\end{tabular}

and the strong Kleene interpretation of the quantifiers, where $X$ is a set of truth values:

$$
\forall(X)=\min (X) \exists(X)=\max (X)
$$

Definition 2 A Kleene-Kripke model for a language $\mathcal{L}$ is a pair $\langle D, I\rangle$ where $D$ is a domain of elements such that $\mathcal{L}_{\text {base }} \subseteq D$ and $I$ is an interpretation such that:

- $\quad$ for a term $t, I(t) \in D$; for tuples $\vec{t}=\left(t_{0}, \ldots, t_{n-1}\right), I(\vec{t})=\left(I\left(t_{0}\right), \ldots, I\left(t_{n-1}\right)\right)$

- where $\ulcorner\varphi\urcorner$ is a distinguished name for $\varphi, I(\ulcorner\varphi\urcorner)=\varphi$

- for an $n$-ary predicate $R, I(R)$ maps $n$-tuples from $D^{n}$ to $\left\{0, \frac{1}{2}, 1\right\}$

- for atoms $R\left(t_{0}, \ldots, t_{n-1}\right)$, with $R$ an $n$-ary predicate, $I\left(R\left(t_{0}, \ldots, t_{n-1}\right)\right)=$ $I(R)\left(I\left(t_{0}\right), \ldots, I\left(t_{n-1}\right)\right)$ 
- sentential connectives and quantifiers are given the strong Kleene interpretation above

- $\quad$ for a truth predicate $T, I(T\ulcorner\varphi\urcorner)=I(\varphi)$ for all formulae $\varphi$

As usual, a Kleene-Kripke model induces a valuation $v$ that can be recursively extended through the language.

Then we define strict-tolerant validity, first introducing some preliminary definitions:

Definition 3 An ST-counterexample to a sequent $[\Gamma>\Delta]$ is a Kleene-Kripke valuation $v$ such that $v[\Gamma] \cap\left\{0, \frac{1}{2}\right\}=\varnothing$ and $v[\Delta] \cap\left\{\frac{1}{2}, 1\right\}=\varnothing$.

To give meaning to this, consider the interpretation of the value $\frac{1}{2}$ as a "paradoxical" value. A standard example of such a use would be assigning a Liar sentence $\frac{1}{2}$ as an indicator that the sentence is both true and false.

Given such an interpretation, we can consider the verification of a formula $\varphi$ in two modes: One may be concerned with establishing that $\varphi$ is true and only true-a strict verification-corresponding to $v$ assigning $\varphi$ the value 1 . But one could take a more relaxed approach, accepting $v$ as a tolerant verifier in case $\varphi$ is at least true, although it may yet hold that $\sim \varphi$ is likewise true.

In other words, $v$ is an ST counterexample to a sequent when it strictly verifies every formula in the antecedent but fails to tolerantly verify any member of the succedent.

If $v$ does not serve as a counterexample to a sequent, we consider it a verifier of that sequent. To make matters more explicit, we make this precise:

Definition 4 An ST-verifier of a sequent $[\Gamma>\Delta]$ is a Kleene-Kripke valuation $v$ such that either $v[\Gamma] \cap\left\{0, \frac{1}{2}\right\} \neq \varnothing$ or $v[\Delta] \cap\left\{\frac{1}{2}, 1\right\} \neq \varnothing$.

The strict-tolerant intuition of ST is manifested in the corresponding notion of validity, according to which $[\Gamma \succ \Delta]$ is valid when any valuation that strictly verifies each formula in $\Gamma$ must tolerantly verify some formula in $\Delta$.

More precisely:

Definition 5 We say that a sequent $[\Gamma>\Delta]$ is ST-valid, and write this $\vDash_{\mathrm{ST}}[\Gamma>\Delta]$ if for all Kleene-Kripke valuations $v$, if $v[\Gamma]=\{1\}$, then $v[\Delta] \cap\left\{\frac{1}{2}, 1\right\} \neq \varnothing$.

The definition of ST-validity clearly can be recapitulated in multiple ways. For example, we could just as easily have stated that $\vDash_{S T}[\Gamma \succ \Delta]$ if $[\Gamma \succ \Delta]$ has no ST-counterexamples.

\subsection{ST and Classical Logic}

Classical validity for the $T$-free fragment of the language is preserved in ST, since every classical countermodel can be extended into an ST-countermodel and every ST-countermodel immediately becomes a classical countermodel. Furthermore, the 
preservation of classical validity for the $T$-free fragment remains unaffected even when the full language is under consideration. In other words, the collection of classically valid $T$-free inferences is included in the collection of ST-valid inferences. ${ }^{1}$

Example 1 The sequent $[\varphi>\varphi]$ is ST-valid. For any Kleene-Kripke valuation $v$, if $v(\varphi)=1$, it trivially follows that $v(\varphi) \in\left\{\frac{1}{2}, 1\right\}$, so there can be no ST-counterexample to $[\varphi>\varphi]$.

Critically, ST is non-transitive in the sense that in general the derivability of sequents $[\Gamma \succ \varphi]$ and $[\varphi \succ \Delta]$ is insufficient to establish the derivability of $[\Gamma \succ \Delta]$, i.e., the rule Cut

is not admissible.

$$
\frac{[\Gamma \succ \Delta, \varphi] \quad\left[\varphi, \Gamma^{\prime} \succ \Delta^{\prime}\right]}{\left[\Gamma, \Gamma^{\prime} \succ \Delta, \Delta^{\prime}\right]}
$$

The case of a formal Liar sentence $\lambda$ - e.g., for a $\lambda=\sim T(\ulcorner\lambda\urcorner)$-provides a further opportunity for an illustration of the properties of ST.

Example 2 The sequents $[\lambda>]$ and $[-\lambda]$ are ST-valid, although the empty sequent $[\succ]$ is not. The semantics guarantee that for any valuation $v, v(\lambda)=\frac{1}{2}$, i.e., although $\lambda$ has no strict verifiers, it is nevertheless tolerantly verified by every $v$. These two facts establish the validity of $[\lambda \succ]$ and $[\succ \lambda]$, respectively. However, because $v[\varnothing]=\varnothing$, every $v$ is an ST-counterexample to $[>]$.

The fact that ST is a conservative extension of CL has been received with varied degrees of optimism. One of ST's biggest selling points is that it can be used to develop a theory able to accommodate a fully transparent truth predicate. And since there is no need to forfeit any classically valid inference, there is also no need to enhance the resulting theory with "the 'classical recapture' that so exercises many non-classical theorists. If the classical bird is never let out of the cage in the first place, there is no need to recapture it" [17].

But even if arguments such as Ripley's merely imply some degree of closeness between ST and classical logic, the conclusion that they are identical is but a few steps away. The fact that ST collects every classically valid inference while adding no new ones into the mix, plus the fact that a well-known criterion for the identity of logics is that they share the same collection of valid inferences yields the (un)desirable conclusion that ST is classical logic.

Several groups of logicians have recently launched similar challenges to the assertion that the "classical bird" has not flown. On the one hand, the Cagliari plan [8] includes an argument to the effect that ST most definitely is not classical logic, it is indeed (very close to) LP. On the other hand, the Buenos Aires plan was born out of a more skeptical stance: They refuse to identify ST with either LP or classical logic, and offer a novel criterion for the identity of logics that gives some traction to their conclusion. We will reconstruct each of their criticism in turn.

\footnotetext{
${ }^{1}$ For a detailed exposition of these results, see [7, pp. 847-853].
} 
Dicher and Paoli [8] point out that, besides attributing the same collection of valid inferences to ST and classical logic, another maneuver that Cobreros, Egré, Ripley and van Rooij rely on to make "things appear reassuringly classical" is choosing a standard sequent calculus presentation for classical logic without Cut as the prooftheoretic presentation for ST. Thus, Dicher and Paoli can level a double criticism to the identification of ST with classical logic: not only does ST present failures of classically valid inferences, but the sequent calculus system chosen by Cobreros et al is shown to be "badly incomplete" with respect to the valid metainferences of ST. 2

By providing a complete proof-theoretic presentation of ST, Dicher and Paoli are able to highlight a major difference between ST and classical logic: there is a piece of vocabulary - to wit, the constant $\lambda$-which classical logic deems as having a bad inferential role while ST judges its role as a good one. Thus, ST cannot be classical logic, since it accommodates a piece of vocabulary known to non-conservatively extend classical logic. Furthermore, they recognize that the reason why $\lambda$ conservatively extends ST but not classical logic is because ST does away with the unrestricted validity of Cut. In this regard, Barrio and his collaborators consider that the unrestricted validity of Cut is an essential component of classical logic. Moreover, their proposal for a criterion to evaluate the identity of logics consists in taking into consideration every single metainferential level instead of restricting oneself to the collection of valid inferences (between formulas). In other words, rather than only looking at inferences which tell us which formulas follow from others, we should also be looking at inferences which tell us what inferences follow from other inferences, and so on. ${ }^{3}$ At the core of their approach lies the following observation:

For classical logic, there is no difference between validity and external validity. Every valid argument is externally valid and viceversa. It turns out that for substructural logics such as ST these two notions no longer coincide.[2, pp. 555$556]$

To make sense of this observation, we begin by recovering the notions of internal and external validity from [1, p. 163] and [13, p. 451]:

Let $\mathbf{S}$ be a sequent calculus system over a language $\mathcal{L}$. Then, a sequent $[\Gamma>\Delta]$ is

- a sequent $[\Gamma \succ \Delta]$ is internally valid iff $[\Gamma>\Delta]$ is a provable sequent of S. As Mares and Paoli note, this defines a multiset consequence relation for any calculus that proves all instances of $[\varphi>\varphi]$ and in which Cut is at least admissible.

- A sequent $[\Gamma>\varphi]$ is externally valid iff $[>\varphi]$ is provable once the sequents $\left[>\psi_{1}\right], \ldots,\left[\succ \psi_{n}\right]$ for each $\psi \in \Gamma$ are added to the system ax axioms and the rule Cut is taken as primitive.

We are now prepared to explain the core observation from the Buenos Aires plan. Since primitiveness implies admissibility, classical logic has Cut as a primitive rule

\footnotetext{
${ }^{2}$ The emphasis is theirs.

${ }^{3}$ The key idea here is that, for any inferential level $n$, there is an inferential level $n+1$ which takes inferences $_{n}$ as its premises and conclusions.
} 
and then takes advantage of its admissibility by allowing us to do without the applications of Cut in the proof of any classically valid sequent. Thus, as long as we begin from the assumption that Cut is primitive, as in the Buenos Aires plan, external validity and internal validity will match. However, if we begin from the admissibility of Cut and build up from there, we will find that, if we want to validate any sequent built out from the formulas of an $\mathcal{L}$ that contains $\lambda$, Cut cannot be primitive in said system, and so internal validity will not match external validity.

Thus, even if classical logic coincides with ST at the level of validity, they differ with respect to what they take to be externally valid. With this in mind, the Buenos Aires plan's proposal to enrich the traditional criterion to include what is counted as externally valid by each logic seems quite reasonable. Moreover, a generalization of their proposal is within reach: If two logics $\mathbf{L}_{\mathbf{1}}$ and $\mathbf{L}_{\mathbf{2}}$ differ at some meta inferential level, then $\mathbf{L}_{\mathbf{1}} \neq \mathbf{L}_{\mathbf{2}}$. We will not stop to evaluate the merits of this new criterion for the identity of logics. Rather, we will simply point out that, although both the Buenos Aires and the Cagliari plan take different roads to reach the conclusion that ST and classical logic are not identical, both proposals are built upon the same fact: the non-transitivity of ST.

\subsection{The Role of Bounds Consequence}

Ripley's argument against Cut in [17] presupposes a bounds consequence interpretation of sequents. As described by Greg Restall in [15], a sequent $[\Gamma>\Delta]$ is interpreted as a position or state that may be adopted by an agent.

In what follows, we will use the notion of a state. Given a particular language,... a state expressed in that language is a pair of sets of statements expressed in that language... A state might be used to represent the outlook of an agent which we take to accept each statement in $\Gamma$ and reject each statement in $\Delta$. We might also use a state to represent the context in some dialogue or discourse at which each statement $\Gamma$ is asserted and each statement in $\Delta$ is denied.[15]

According to this interpretation, the derivability of a sequent corresponds to proscription against taking the corresponding position, i.e., that the position is outof-bounds in the sense that it would violate some norm. Notably, insofar as bounds consequence places acceptance and rejection on equal footing, it may be thought of as a bilateralist theory.

The consequent interpretation of Cut is an extensibility condition, i.e., that for any statement $\phi$, either it is in-bounds to assert $\phi$ or it is in-bounds to deny $\phi$. In the case of e.g. a Liar sentence, it is out-of-bounds both to assert and to deny the statement; neither is an acceptable position to take.

The necessity of the failure of Cut can be easily seen by observing that-if we take $[\Gamma-\Delta]$ to mean that the position that asserts everything in $\Gamma$ and denies at least one member of $\Delta$ is out of bounds—when $\lambda$ is the formula $\sim T(\ulcorner\lambda\urcorner$ ) (that is, the Liar), we have the following: 
1. Every position $[\Gamma>\lambda]$ is out of bounds, since denying $\lambda$ is always out of bounds. As a result, $[\Gamma>\lambda]$ is valid. This is of special importance in those cases in which asserting every member of $\Gamma$ is in bounds.

2. Since asserting $\lambda$ is always out of bounds, there is no way to be in a position $[\lambda \succ \Delta]$ such that it is in bounds. In particular, seeing that we are already out of bounds when asserting $\lambda$, we can deny every member of $\Delta$ without any major consequence, even when it is not out of bounds to deny the elements of $\Delta$. Thus, for all $\Delta,[\lambda>\Delta]$ is valid.

As a consequence of the latter two facts, we can see that unrestricted applications of Cut would lead us to believe that no position which asserts every member of $\Gamma$ but denies every member of $\Delta$ is ever in-bounds, which would entail the validity of any sequent $[\Gamma>\Delta]$ whatsoever. Thus, as we already saw above, in order to avoid this undesirable result, the validity of Cut has to be restricted.

Both groups of logicians remark upon another important fact that follows from ST's substructurality: that each of the elements in the collection of ST-invalid metainferences have a counterpart in the collection of LP-invalid inferences. For example, instances of the metainferences meta Modus Ponens

$$
\frac{[\ulcorner\varphi] \quad[>\varphi \supset \psi]}{[\ulcorner\psi]}
$$

and meta Explosion

$$
\frac{[\succ \varphi] \quad[>\sim \varphi]}{[\ulcorner\psi]}
$$

described in [2] are invalid every time $\varphi$ is set to $\lambda$ and $\psi$ is set to any formula such that $v(\psi)=0$. This leads Dicher and Paoli to embrace the closeness between ST and LP, arguing that there is very little room to deny that ST and LP are indeed the same logic. According to the Buenos Aires plan, one can genuinely resist the claim that ST can be identified with either classical logic or with LP. ${ }^{4}$

What is most important about this common ground is that it allows the expression of the criticism against ST as classical logic within the bounds consequence framework. An important piece in Ripley's strategy is that Cut need not be an essential component of the bilateralist framework. He relates this to the eliminativity of Cut in sequent proof systems for classical logic: we do not need to make any special provisions for Cut, since the set of classically valid arguments is already closed under it. Likewise, it might be that assertion and denial do not depend on any vital way on the availability of Cut; rather, it might just be that assertion and denial are transitive when one restricts oneself to classical vocabulary.

Just as Barrio and his collaborators take Cut to be an essential component of classical validity, so too has Greg Restall argued for Cut as vital for assertion and denial in [15]. If Restall were right then, contra Ripley, the logic of assertion and denial would match classical logic in that it too would be closed under Cut at the metainferential level.

\footnotetext{
${ }^{4}$ The discussion of why ST is not classical logic can be found in [3, p. 114]. Moreover, for the rejection of the idea that ST is LP, see [3, pp. 116-8].
} 
As we saw, bounds consequence is crucial because sentences whose assertion and denial are both out of bounds are supposed to be an expected component of the framework. But this is not a framework like the one Restall has in mind, in which out-of-bounds assertion corresponds with in-bounds denial and viceversa. Such a framework includes the assumptions that (1) assertion corresponds with truth and denial with untruth, and (2) assertion and denial are mutually exclusive. Given all of this, Liar sentences would be as non-conservative in this kind of bilateralist context as they are in plain classical logic, since we would be forced to both assert and deny $\lambda$, breaking our initial assumptions.

Notice that the above paragraph contains no explicit mention of Cut or transitivity for assertion or denial, and still, we have not been able to accommodate $\lambda$ without breaking one of our starting assumptions. Here, as Barrio et al. point out, the parallel between Cut being non-essential for assertion and denial and the eliminability of Cut in some proof systems break down. Even if we did not specify anything about transitivity in a bilateralist framework, it almost appears as if it were already baked into the framework.

We are now back at the idea (introduced in Section 2.2) that the explicit mention of Cut can be eliminated when proving classical validities because in some sense the rule is already there. This is why sentences such as the Liar become non-conservative extensions of classical logic. In contrast, Cut seems to be merely admissible in ST for completely different reasons: it seems like we are able to help ourselves to just enough Cut to coincide with classical logic but then stop. That we are able to do this is due to the distinction between strict and tolerant assertion and denial, which, as Ripley rightfully points out, collapse in the presence of Cut. If, as we have stated, Cut is eliminable because it was already there, then it is unsurprising that classical logic would be unable to distinguish between strict and tolerant acts.

But how could we be able to decide just how much Cut is enough? Dicher and Paoli argue that an important role of $\top$ and $\perp$ is to encode strict assertion and denial, respectively. Their claim is then that although Ripley is right in construing tolerant assertion/denial as "silent partners" of their strict counterparts, he fails to notice that classical logic has no way of distinguishing between both kinds of attitudes. For them, $\lambda$ is the mark of tolerant attitudes, which is to say, it is the signal that an application of Cut is no longer safe. Without this indication, classical logic "would happily cut all over the place, which, under appropriate circumstances, has disastrous effects" [8].

\section{Strict-Tolerant Hierarchies}

Regardless of where one stands on the issue of identifying ST with LP, there is an agreement between both sides of this particular issue regarding the fact that ST has simply pushed non-classicality up one level, to the level of metainferences. More importantly, we must recall that the Buenos Aires proposal for a new criterion for the identity of logics involves considering every inferential level, not just the first two. This is because they have proven a result to the effect that "it is possible to obtain a sequence of logics which can be progressively [i.e., linearly] ordered in terms of their degree of classicality" [3]. 
To understand what the components of the hierarchy are, we will begin by recalling that a sequent $[\Gamma>\Delta]$ is an ordered pair $(\Gamma, \Delta)$ of finite, possibly empty subsets of $\mathcal{L}_{\text {base }} S E Q_{0}\left(\mathcal{L}_{\text {base }}\right)$ is the set of all such sequents. Sequents are typically intended to encode inferences, arguments or some such relation between a set of formulas which encode premises and a further set which encodes conclusions. Metainferences are then inferences between sets of inferences and are thus encoded by metasequents (sequents of sequents).

Formally, metainferences of the first level and onwards can be recursively defined as follows: $S E Q_{n}\left(\mathcal{L}_{\text {base }}\right)$ is the set of sequents $[\Gamma>\Delta]$, which in turn are understoood as ordered pairs $(\Gamma, \Delta)$ of finite, possibly empty subsets of $S E Q_{n-1}\left(\mathcal{L}_{\text {base }}\right)$. Then, the set of metainferences is the set of ordered pairs $(\Gamma, \Delta)$ of finite, possibly empty subsets of $S E Q_{0}\left(\mathcal{L}_{\text {base }}\right)$. Rules from a sequent calculus are the paradigmatic examples of metainferences. Frequently, we wish to distinguish metainferences from sequents, whence a metainference of level $n$ will often be represented as $\Gamma \Rightarrow \Phi$ where $\Gamma \cup\{\Phi\}$ is a set of metainferences of level $n-1$.

Different sources have informally defined metainferences as

- schematic principles about the consequence relation, which have inferences as instances [cf. Barrio et al., [2], pp. 555-7]

- "[...] syntactic objects of the system under consideration", [8]

- " $[\ldots .$.$] closure properties on the set of valid arguments [16], or$

- "[...] principles under which a consequence relation might or might not be closed". [7].

Although we are partial to construing metainferences as syntactic objects rather than schemas, we will not go into the details behind this choice. It will suffice to say that construing inferences as syntactic objects makes it easier to give an uniform treatment to all levels of the hierarchy.

\subsection{The Logic TS}

The notion of strict-tolerant consequence has tolerant-strict consequence as its dual, yielding the logic TS described by French in [11]. As a dual to ST, one can characterize TS by appeal to tolerant-strict counterexamples to a sequent, i.e., valuations $v$ that tolerantly verify every member of the antecedent but strictly verify no member of the succedent. Explicitly, this is:

Definition 6 A TS-counterexample to a sequent $\Gamma>\Delta$ is a Kleene-Kripke valuation $v$ such that $v[\Gamma] \subseteq\left\{\frac{1}{2}, 1\right\}$ and $1 \notin v[\Delta]$.

As before, all Kleene-Kripke valuations that are not TS-counterexamples for a sequent are considered its TS-verifiers.

Definition 7 A TS-verifier of a sequent $\Gamma \succ \Delta$ is a Kleene-Kripke valuation $v$ such that either $0 \in v[\Gamma]$ or $1 \in v[\Delta]$. 
Definition 8 We say that a sequent $[\Gamma>\Delta]$ is TS-valid and write this $\vDash_{\mathrm{TS}}[\Gamma>\Delta]$ if $[\Gamma \succ \Delta]$ has no TS-counterexamples.

A few facts about this: For one, we can notice that there are no valid sequents in TS. For any signature $\sigma$ there is a Kleene-Kripke model that maps all atoms-and hence, all complex formulae-to the value $\frac{1}{2}$, Hence, for all sets of formula $\Gamma \cup \Delta$, there is a $v$ such that $v[\Gamma \cup \Delta] \subseteq\left\{\frac{1}{2}\right\}$. Such a $v$ will serve as a TS-counterexample to the sequent $[\Gamma>\Delta]$; as $\Gamma \cup \Delta$ was arbitrary, there are no valid sequents.

Although the discussion of TS in [11] is interesting, our interest in TS is primarily limited to the role it plays in the definition of systems in the hierarchy.

\subsection{Constructing Hierarchies}

Having complemented the notion of strict-tolerant evaluation with the notion of tolerant-strict evaluation up to the level of metainferences, one may generalize the intuitions as shown in e.g., [2] and [3].

The characteristic features of strictness and tolerance with respect to verifiers as described above can be captured by appeal to the level of permissivity each brings to verification. A tolerant verifier is more liberal in which formulae it affirms-e.g., $\sim T(\ulcorner\lambda\urcorner)$ — than its more conservative relative. Yet one can be more or less permissive not only to validity of a position, but also to the validity of a metainference, providing a suitable anchor point on which to focus a generalization.

Crucially, we may observe that ST is more permissive than TS in the sense that ST verifies the validity of more positions than its cousin. This permissivity shows that strict-tolerant validity is itself a tolerant notion. Conversely, inasmuch as TS has no validities, its account of validity is maximally strict, whence it is appropriate to consider TS validity as a strict notion.

We formulate the technique described in [3] for generating metainferential logics in the hierarchy:

Definition 9 We take the notions of strictness and tolerance and generalize them to an infinite hierarchy. Let us take the following definitions:

- Strict $_{1}=\mathrm{TS}$, and

- Tolerant $_{1}=\mathrm{ST}$

Then for each $n \in \omega$, we define a tier:

- Strict $_{n+1}=$ Tolerant $_{n} /$ Strict $_{n}$, and

- Tolerant $_{n+1}=$ Strict $_{n} /$ Tolerant $_{n}$

For example, TSST is, per this definition, Strict $1 /$ Tolerant $_{1}=$ Tolerant $_{2}$.

As a referee has observed, Definition 9 assumes that each stage follows either a strict-tolerant or tolerant-strict paradigm. But just as truth-functional semantics for Priest's LP reveal that it could be considered a tolerant-tolerant logic, one could evaluate metainferences as either tolerant-tolerant or strict-strict. Thus, the 
uniformity presupposed in the definition rules out many available permutations of metainferential logics, e.g., the metainferential logic STST.

While the definition is restrictive in this way, the metainferential logics Definition 9 covers enjoy a sort of stability absent in those outside its scope. In each system it defines, inferences of all stripes - metainferences of all levels-follow a uniform pattern: Either metainferences are guided by a strict-tolerant principle or they are guided by a tolerant-strict principle. Of all these metainferential logics, the ones interesting to us are those that endorse a thesis that all inferences-metainferences of any level-are strict-tolerant. As each of these systems is covered by Definition 9, it is broad enough in its scope for our purposes, at least.

The definitions of [3] can be further recast as follows, starting with TSST and STTS as bases:

Definition 10 An TSST-countermodel for an inference $\left\{\left[\Gamma_{0}>\Delta_{0}\right], \ldots,\left[\Gamma_{n}-\right.\right.$ $\left.\left.\Delta_{n}\right]\right\} \Rightarrow[\Xi \succ \Theta]$ is a Kleene-Kripke valuation $v$ such that:

- $\quad v$ is a TS-verifier for every sequent $\left[\Gamma_{i}>\Delta_{i}\right]$ for $i \leq n$

- $v$ is an ST-counterexample for the sequent $[\Xi \succ \Theta]$

Definition 11 An STTS-countermodel for an inference $\left\{\left[\Gamma_{0}>\Delta_{0}\right], \ldots,\left[\Gamma_{n} \succ\right.\right.$ $\left.\left.\Delta_{n}\right]\right\} \Rightarrow[\Xi \succ \Theta]$ is a Kleene-Kripke valuation $v$ such that:

- $\quad v$ is a ST-verifier for every sequent $\left[\Gamma_{i}>\Delta_{i}\right]$ for $i \leq n$

- $v$ is an TS-counterexample for the sequent $[\Xi>\Theta]$

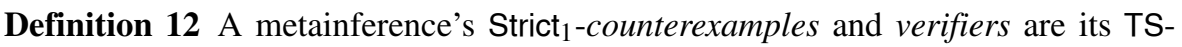
counterexamples and verifiers, respectively (described in Definition 3).

Definition 13 A metainference's Tolerant ${ }_{1}$-counterexamples and verifiers are its STcounterexamples and verifiers, respectively (described in Definition 6).

These provide the bases for counterexamples and verifiers which may be extended:

Definition 14 We define Strict $_{n}$ and Tolerant ${ }_{n}$ counterexamples and verifiers by simultaneous induction. Definitions 12 and 13 give the case in which $n=1$. For $n>1$, we employ the following:

- A Strict $_{n}$-counterexample for a metainference $\left\{\Phi_{0}, \ldots, \Phi_{n}\right\} \Rightarrow \Phi$ is a KleeneKripke valuation $v$ such that:

- $\quad v$ is a Tolerant ${ }_{n-1}$-verifier for each $\Phi_{i}$

- $\quad v$ is a Strict $_{n-1}$-counterexample for $\Phi$

A Strict ${ }_{n}$-verifier for a metainference is any valuation $v$ that is not a counterexample to it.

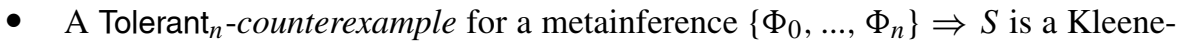
Kripke valuation $v$ such that:

- $\quad v$ is a Strict $_{n-1}$-verifier for each $\Phi_{i}$ 
- $\quad v$ is a Tolerant ${ }_{n-1}$-counterexample for $\Phi$

A Tolerant ${ }_{n}$-verifier for a metainference is any valuation $v$ that is not a counterexample to it.

Just as metainferences (evaluated by TSST or STTS) are defined as collections of inferences imbued with a particular structure-the types of objects evaluated by TS or ST-the objects evaluated by the inferences at some level in the hierarchy will be sets of objects at the previous level.

Barrio and his collaborators have indicated in conversations that they view metainferences of any of such metainferential logics to be non-linguistic in nature. In other words, the Buenos Aires plan resists describing the metainferences considered by e.g., TSST as formulas. But, certainly, a metainference such as

$$
\text { If }[>\varphi] \text { and }[>\varphi \supset \psi] \text { then }[>\psi] \text {. }
$$

is correlated with readily-identifiable and precise linguistic description (whence metainferences can be described at least metalinguistically) and is constructed according to recursive processes. These facts, we feel, provide adequate grounds for formally describing the class of metainferences of any particular tier as a formal language. We make this precise by describing an infinite collection of languages.

Definition 15 The languages $\mathcal{L}_{i}$ for the following purposes will be defined as follows:

- $\mathcal{L}_{0}=\mathcal{L}_{\text {base }}$

- $\quad \mathcal{L}_{1}=\left\{[\Gamma>\Delta] \mid \Gamma, \Delta \subset \mathcal{L}_{0}\right\}$

- for $i>1, \mathcal{L}_{i}=\left\{\left\{\varphi_{0}, \ldots, \varphi_{n-1}\right\} \Rightarrow \psi \mid\left\{\varphi_{0}, \ldots, \varphi_{n-1}, \psi\right\} \subseteq \mathcal{L}_{i-1}\right\}$

\section{The Limitations of the ST Hierarchy}

The notion of a metainferential logic is noteworthy for its making explicit that metainferences, too, are subject to their own canons of reason to which we may apply formal techniques. Moreover, we believe that, if one accepts the generalized ST thesis that inference in general is best understood by a strict-tolerant interpretation, any member of the ST hierarchy describing the inferential interactions holding between metainferences of a particular tier correctly captures those interactions.

However, we believe that there exist several glaring defects in the scope of any of the metainferential logics lying in the hierarchies described by Barrio and his collaborators - whether they are in the ST hierarchy, the TS hierarchy, or otherwise. In this section, we will identify several difficult features of the general approach that are critical obstacles to its application and conceptual soundness. Roughly, these can be described as:

- The regimented nature of the hierarchy's operands excludes many obviously "metainferential" arguments used in practice.

- The nature of its construction hinders the definition of a limit to the hierarchy. 
- As one moves up the hierarchy, the semantics swiftly (almost immediately) becomes impractical

We will consider these three points in order over the course of the following subsections.

\subsection{Mixed Inferences}

As we have noted, the regimentation of the languages treated by any of these hierarchies is extremely strict - a metainferential logic of tier $n+1$ takes as its objects only inferences of tier $n$-and the severity of this regimentation entails a commensurately severe restriction to the logic's expressivity.

Two consequences are especially important: First, note that the language treated by a metainferential logic is disjoint with the language of the previous tier, e.g., while the construction of $\mathcal{L}_{n+1}$ is defined in terms of $\mathcal{L}_{n}, \mathcal{L}_{n+1}$ is not its closure. Thus, there is no common language that purports to describe metainferences in toto. That $\mathcal{L}_{m}$ and $\mathcal{L}_{n}$ are pairwise disjoint whenever $m \neq n$ complicates the matter of defining a hierarchy's limit. The disjointedness means that a limit cannot be obtained by iterative applying an operation to the base class and finding a fixed point. The alternativethat the inferences serving as the limit's operands are infinitary objects-seems implausible. This matter, we will argue, can be satisfactorily resolved by a solution to the second consequence, which we now treat in more detail.

The second consequence, we suggest, is that the sharp limitations to the expressivity of any one of these logics leads to an equally sharp limitation to its applicability. Insofar as only metainferences from a single tier $n$ are considered by a logic in a hierarchy, one cannot straightforwardly represent metainferences of distinct tiers in the same setting, much less correctly characterize any consequences which may be inferred from their joint validity. The impoverishment of the individual systems' expressivity proscribes from consideration an incredibly rich and natural range of natural inferential practices, namely, inferences that take as premises metainferences of distinct tiers. We will call such inferences mixed inferences. ${ }^{5}$

A cursory appraisal of human inferential practices reveals that mixed inferences are incredibly prevalent. Frequently, as logicians and philosophers, we unabashedly appeal to both inferential and metainferential principles concurrently. The following, for example, seems to be a perfectly coherent argument form:

The sentence $\varphi$ is a theorem. Moreover, it holds that whenever $\varphi$ is a theorem, $\psi \supset \varphi$ will, too, be a theorem. Thus, $\psi \supset \varphi$ is a theorem.

Obviously, not all will accept the validity of this argument; many relevant logicians will disagree with the second premise. However, even the relevant logician will recognize the above as an intelligible principle that can be resisted. In other words, the intelligibility of the assertion is not diminished for its appeal to both inferential and metainferential principles in tandem.

\footnotetext{
${ }^{5}$ Formally, we include inferences whose premises are drawn from the same tier as degenerate cases, although we will often consider cases to be "authentically" mixed when making a rhetorical point.
} 
Indeed, in formal practice, we assert the validity of such mixed inferences ceaselessly. A proof theorist proving e.g. Cut elimination for some sequent calculus, for example, may appeal to provable sequents, admissible rules, and known patterns governing which rules are admissible in the same proof. Similarly, if a modal logician establishes both that a sentence is true in each member of a class of Kripke models and that rule necessitation-recognizably a metainference-holds for this class, these facts may be fruitfully applied to infer that other sentences are true in these models.

Let us fix a particular (semi-formal) mixed inference to serve as an illustration. Intuitively, despite its appeal to multiple tiers, the following is an intelligible (if informal) argument:

The sequent $[>\varphi]$ is valid and the rule

$$
\frac{[\Gamma \succ \varphi, \Delta]}{[\Gamma \succ \varphi \vee \varphi, \Delta]}
$$

is correct. Therefore the sequent $[-\varphi \vee \varphi]$ is valid.

To ensure that we accurately consider such arguments we recall our earlier definition of formal languages for metainferences and provide a formal language $\mathcal{L}_{\text {mix }}$ of mixed inferences.

Definition 16 The language $\mathcal{L}_{\text {mix }}$ of mixed inferences is the set

$$
\mathcal{L}_{1} \cup\left\{\left\{\Phi_{0}, \ldots, \Phi_{n-1}\right\} \succ \Psi \mid\left\{\Phi_{0}, \ldots, \Phi_{n-1}, \Psi\right\} \subseteq \bigcup_{i \in \omega} \mathcal{L}_{i}\right\}
$$

Our foregoing example of a mixed inference may serve to illustrate $\mathcal{L}_{\text {mix }}$ by showing its correlate in the formal language. This metainference is captured by the formula:

$$
\{[>\varphi],\{[\Gamma>\varphi, \Delta]\} \Rightarrow[\Gamma>\varphi \vee \psi, \Delta]\} \Rightarrow[>\varphi \vee \psi]
$$

One might object that appealing to examples of practical uses of mixed inferences at relatively low levels doesn't establish a need for mixed inferences at arbitrarily high levels. Surely, there is some relatively low level of the hierarchy such that no human reasoner has yet made use of a mixed inference involving this tier. But a theory of inference clearly should not limit itself only to inferences that have been expressed historically but should be expressive enough to capture all inferences that can be expressed. Classical logic still governs inferences between formulae with millions of atoms even if no human will ever make such an expression; this case is no different.

As an analogy, consider that for any formal programming language there is a relatively small number for which the syntactic complexity of any expression in use does not exceed that threshold. But this does not mean that the formal definition of the language can stop at this threshold, for a need may arise for an expression of a slightly greater complexity than this. Likewise, it is not that in practice we presently use mixed inferences of arbitrarily high tiers that demands that $\mathcal{L}_{\text {mix }}$ includes mixed inferences across any tiers; rather, the demand follows from the fact that we can employ them — and can expect to find them intelligible — if the need should arise. 
We now consider a potential rejoinder to our charge of inadequate expressivity, namely, that on translation, any inference or metainference of $\mathcal{L}_{m}$ can be translated to a corresponding metainference of $\mathcal{L}_{n}$ for any $n>m$. Thus, by encoding the premises and conclusion of any mixed inference so that their translations uniformly lie in a $\mathcal{L}_{j}$, the putatively mixed inference itself thereby enjoys a representation in $\mathcal{L}_{j+1}-$ a statement describing an inferential relation between metainferences of $\mathcal{L}_{j}$-which may be analyzed by an appropriate logic in the ST hierarchy.

To provide a simple illustration of the suggestion, the sequents $[>\varphi]$ and $[-\varphi \vee \psi]$ from the above example are members of $\mathcal{L}_{0}$ while the metainference $\{[\Gamma>\varphi, \Delta]\} \Rightarrow[\Gamma>\varphi \vee \psi, \Delta]$ is a member of $\mathcal{L}_{1}$. The rejoinder suggests that the informal mixed argument is adequately captured by first translating the $\mathcal{L}_{0}$ objects [ $-\varphi]$ and $[-\varphi \vee \psi]$ to corresponding $\mathcal{L}_{1}$ objects \{\}$\Rightarrow[-\varphi]$ and \{\}$\Rightarrow[>\varphi \vee \psi]$, respectively. As the premises and conclusion are all in $\mathcal{L}_{1}$, the regimented definition of $\mathcal{L}_{2}$ includes

$$
\{\{\} \Rightarrow[>\varphi],\{[\Gamma>\varphi, \Delta]\} \Rightarrow[\Gamma>\varphi \vee \psi, \Delta]\} \Rightarrow\{\} \Rightarrow[\quad>\varphi \vee \psi]
$$

the validity of which can be evaluated against $e . g$. TSST.

There are, we admit, some elements of this position that are compelling. On one reading, our example's taking the sequent $[>\varphi]$ as a premise was tantamount to hypothesizing its validity. And, in such cases, it may seem plausible to equate the validity of a sequent $[>\varphi]$ with the admissibility of the rule

$$
\overline{[-\varphi]}
$$

After all, one might say, there is nothing to validity of a sequent in a proof system beyond its derivability from any arbitrary set of premises.

The outward equivalence between a metainference and its translation wears thin, however, as we consider more general cases. Considering such cases reveals that this technique too swiftly identifies objects that are clearly conceptually dissimilar. Intuitively, this is simple enough: The two assertions are about different things and differ in subject matter. To claim that something holds is obviously different than to claim that all inferences to it are valid; were they not distinct, then the relevant logician could not embrace the validity of $\varphi \vee \sim \varphi$ while resisting the validity of $\psi \rightarrow(\varphi \vee \sim \varphi)$ for arbitrary $\psi$.

Recall that ST rests on the foundation of bounds consequence, according to which the sequent $[-\varphi]$ is read not as " $\varphi$ is a consequence of the empty set," but rather, as "to deny $\varphi$ would be out-of-bounds." Two options exist for interpreting metainferences: One can resist retaining the bounds consequence reading, in which case metainferences are understood operationally, or one can maintain the bounds consequence reading. It seems to us that on either choice, the conceptual distinction between $e . g$. validity of $[>\varphi]$ and the corresponding rule remain clear. We will now examine both of these interpretations.

\subsubsection{The Operational Reading}

If one reads metainferences operationally - in which case the sequent's translation would be read "from any sequent, one may infer the sequent $[>\varphi]$ " - the inference and its translation would seem far less synonymous. One could, after all, 
stipulate the that it is out-of-bounds to deny $\varphi$ (e.g., for argument's sake) without thereby assuming the sequent $[-\varphi]$ to be derivable from all premises.

Furthermore, the operational reading allows for a parallel between the current situation and one involving true formulas and theorems of $\mathcal{L}_{0}$. Recall that in the Buenos Aires approach to the hierarchy, the difference between

- $[\varphi, \varphi \supset \psi>\psi]$ (Modus Ponens, an inference); and

- $\{[>\varphi],[>\varphi \supset \psi]\} \Rightarrow[>\psi]$ (Meta Modus Ponens, a metainference).

is often stressed, especially when arguing against the identity of ST and LP or classical logic.

Some of the most important things that we know about Modus Ponens (MP) and Meta Modus Ponens (mMP) is that although they are both valid in classical logic, they differ in their subject-matter. Andreas Fjellstad puts it nicely when he states that, "While (MP) expresses that the inference from $A \rightarrow B$ and $A$ to $B$ is valid, (mMP) expresses that the inference from the validity of $A$ and the validity of $A \rightarrow B$ to the validity of $B$ is valid in the meta-theory" $[10$, p. 3]. In other words, MP is a schema of inferences that should hold between sentences of the object language, while mMP is a schema that describes a kind of closure for the logical laws which are applied to the object language. ${ }^{6}$

Moreover, we know not to conflate them, it is possible for a logic to be closed under one, but not the other. Non-transitive approaches to logical consequence are an example, but also consider relevant logics, which include a rule form of Modus Ponens (mMP), without necessarily including the axiom form (MP) in their axiomatization (see, for instance [18, p. 287].

There is, however, a point of convergence between both MP and mMP. When evaluating the validity of MP from the supposition that its premises are satisfied, we find that there are two sorts of verifiers: (contingently) true formulas and logical truths. Of these, we know that the latter are precisely the verifiers for the premises of mMP, albeit in a different sense. From the fact that $\varphi$ is a logical truth, two different facts follow:

1. There is a valuation $v$ such that, $v(\varphi)=1$; and,

2. The sequent $[-\varphi]$ has no counterexamples, i.e. $\varphi$ follows from any set of premises.

Consider that, for a logical truth $\varphi$ and a contingent truth $\psi$, there will be at least one model in which they will both be true, namely, there is a (possibly empty) $\Gamma$ such that $\forall \xi \in \Gamma, v(\xi)=1$ and $v(\varphi)=1 / v(\psi)=1$; with $v$ a classical valuation function. The difference will be that it is not the case that not all $\Gamma$ will be such that $\forall \xi \in \Gamma$, $v(\xi)=1$ and $v(\psi)=1$, while it will certainly be the case that for whichever $\Gamma$, $\forall \xi \in \Gamma, v(\xi)=1$ and $v(\psi)=1$. But the latter fact is one about validity, even if it is stated in terms of truth (like the former). Thus, even if two facts are stated in the same terms or treated under the same reading, their subject-matter can still differ.

\footnotetext{
${ }^{6}$ For a related, but opposing, view that at their core MP and mMP share subject-matter and thus should stand or fall together, see [19].
} 
The following quote further advances our point:

(MP) expresses that $\langle\{A \rightarrow B, A\},\{B\}\rangle$ is in $R$ [the validity relation] and (mMP) expresses that if $\langle\varnothing,\{A\}\rangle$ and $\langle\varnothing,\{A \rightarrow B\}\rangle$ are in $R$, then $\langle\varnothing,\{B\}\rangle$ is also in $R$. This means that in the entailment-relation for the meta-language, $R_{M}$, we will find $\langle\{\vdash A, \vdash A \rightarrow B,\{\vdash B\}\rangle[10$, p. 4].

Recall that our preferred definition of the objects in all the levels of the hierarchy was as syntactic objects because it allowed for a uniform treatment. Thus, by treating formulas, sequents and meta ${ }_{n}$ sequents as objects of the same kind, we should expect to be able to look at them from both perspectives. Thus, we should be able to distinguish between the validity of a $\left(\right.$ meta $\left._{n}\right)$ sequent with respect to an arbitrary valuation and its following from the empty set of premise (meta $\left.{ }_{n}\right)$ sequents; that is, its meta-validity expressed in terms of validity.

\subsubsection{The Bounds Consequence Reading}

A drawback of the bounds consequence reading is that it is not meant to be applied to levels above inferences. But then, one of the main benefits of doing this exercise is that it provides an alternative, truly uniform and non-operational reading of inferences at all levels. Taking $[\succ \varphi]$ and \{\}$\Rightarrow[>\varphi]$ as examples, we can roughly say that

- $[-\varphi]$ is stating that "it is (always) out-of-bounds to deny $\varphi$ ", while

- \{\}$\Rightarrow[>\varphi]$ amounts to stating that "it is (always) out-of-bounds to deny that 'it is always out-of-bounds to deny $\varphi$ "”

On this reading, it becomes clear that the two differ in meaning; while $[>\varphi]$ sets a condition about how we should speak about $\varphi,\{\} \Rightarrow[>\varphi]$ sets conditions on how to speak about this condition. An initial concern might be that on the first case, we are (meta-)asserting that "it is (always) out-of-bounds to deny $\varphi$ "; while in the second "it is (always) out-of-bounds to deny that..." might be taken to imply that it is always in-bounds to assert that 'it is always out-of-bounds to deny $\varphi$ '.

Clearly, asserting that denying $\varphi$ is out-of-bounds falls short of an assertion of $\varphi$. Likewise, that it is out-of-bounds to deny that denying $\varphi$ is out-of-bounds falls short of an assertion of "it is (always) out-of-bounds to deny $\varphi$ " unless further assumptions - such as the equivalence between out-of-bounds denial and in-bounds assertion-are taken in. These additional assumptions about assertion and denial would need further justification, and thus would be up for debate.

In the context of intuitionism, the distinction between assertion and out-of-bounds denial is illustrated by an example discussed by Dummett in [9, p. xviii]. Although the formula $\sim \sim(\varphi \vee \sim \varphi)$ (which amounts to denying that bivalence is out-of-bounds due to the existence of counterexamples) is intuitionistically valid, this is a distinct principle than the positive $\varphi \vee \sim \varphi$ (" $\varphi$ or not $\varphi$ "). In other words, the intuitionist's claim that one cannot falsify instances of excluded middle does not necessitate an acceptance of excluded middle itself.

First, we need to relate the bounds consequence reading to intuitionism. We will assume that $[\Gamma>\varphi]$ is read as "it is out of bounds to assert every member of $\Gamma$ and 
deny $\varphi$ " corresponds to the intuitionistic reading that there is a constructive proof of $\varphi$ from $\Gamma{ }^{7}$ If it is not the case that it is out of bounds to assert every member of $\Gamma$ and deny $\varphi$, then there is a proof of $\sim \varphi$ from $\Gamma$.

Thus, the intuitionist should accept the principle that

If the sequent

is valid, and the rule

$$
[\Gamma>\varphi]
$$

$$
\frac{[\Gamma \succ \psi, \Delta]}{[\Gamma \succ \psi \vee \xi, \Delta]}
$$

is valid, then the sequent

$$
[\Gamma \succ \varphi \vee \sim \varphi]
$$

is also valid.

namely,

If one asserts that

$$
[\Gamma>\varphi]
$$

and takes it as out-of-bounds to assert any instance of $[\Gamma>\psi, \Delta]$ while denying the corresponding instance of $[\Gamma>\psi \vee \xi, \Delta]$, then one cannot deny

$$
[\Gamma \succ \varphi \vee \sim \varphi]
$$

We know this is the case because we are zeroing in on the behavior of a specific $\varphi$ in an arbitrary valuation. Thus, there is a $\varphi$ that verifies the first part of the statement-i.e. that the sequent $[\Gamma>\varphi]$ is valid-in virtue of its being either a contingent or a logical truth. Otherwise, the statement will be falsified by the existence of a constructive proof of $\sim \varphi$, guaranteeing that $\varphi$ is not derivable, at least with respect to the arbitrary valuation. Notice that by requiring that the sequent be valid, we are already ruling out potential cases in which the (in)validity of the sequent cannot be determined. Thus, the validity of the argument will be preserved.

In contrast, the following should turn out to be intuitionistically unacceptable:

The validity of the rules

and

$$
\overline{[\Gamma>\varphi]}
$$

$$
\frac{[\Gamma-\psi, \Delta]}{[\Gamma \succ \psi \vee \xi, \Delta]}
$$

entails the validity of the rule

$$
\overline{[\Gamma \succ \varphi \vee \sim \varphi]}
$$

which has the following bounds consequence reading:

If it is out-of-bounds to

1. assert the empty (sequent) set while denying $[\Gamma>\varphi]$; and,

2. assert $[\Gamma \succ \psi, \Delta]$ while denying $[\Gamma \succ \psi \vee \xi, \Delta]$

\footnotetext{
${ }^{7}$ As per usual, $\Gamma$ is possibly the empty set of premises.
} 
then, it is out-of-bounds to assert the empty (sequent) set while denying $[\Gamma>$ $\varphi \vee \sim \varphi]$

That this is unacceptable stems from the fact that, as happened with the illustration of the operational reading above, there is a slight change of meaning that turns this argument into something any intuitionist would not accept, even if they readily admit that they do not have a counterexample at hand. When we switch from the supposition that a particular $\varphi$ may verify or falsify the claim that $[\Gamma \succ \varphi]$ is valid to the general conditions under which such a position would be out-of-bounds-encoded by the metainference from the empty sequent set to the conclusion $[\Gamma>\varphi]-$ we are able to incorporate metainferential generalizations of intuitionistic practice. What we discover is that there will be instances in which we cannot determine the (in)validity of $[\Gamma>\varphi]$ and consequently of the corresponding metainference.

Thus, we will be in a position in which it will be difficult to reject one of the premises, for one would be unable to provide the required proof of $\sim \varphi$. However, the conclusion amounts to stating that it is always out-of-bounds to deny that it is out-ofbounds to assert the empty premise set, and deny that one has either a constructive proof of $\varphi$ or of its negation. Since the intuitionist is open to this possibility, they will be prepared to, minimally, not reject the premises but openly deny the conclusion.

We chose to illustrate the variation of meaning between mixed and uniform inferences using an example from intuitionism because, apart from providing an example of uniformity across metainferences at all levels, the chosen example illustrates a secondary but important point: that although variations of meaning sometimes entail the rejection of one of the versions of an argument (as in the case of the intuitionist), the acceptance of two different versions of an argument does not entail that their meanings coincide. Thus, from the fact that the deep ST-theorist would readily admit two apparently interchangeable versions of an argument need not license the conclusion that the disposition to accept both versions is due to their not seeing a difference in meaning in any of the cases.

\subsection{The Need for a Limit}

If we aim to subsume metainferential practice at an arbitrary level within the hierarchies, there is a need to define a limit within which all such inferences-i.e., inferences of any order-can be described. This is certainly something that classical logic is capable of doing; the character of e.g. metametainferences in classical logic certainly satisfy classical principles.

In [3], Barrio, Pailos, and Szmuc suggest a difficulty with considering the distinction between ST and classical logic to be simply that the two have a metainferential disagreement. After pointing to how the classical fragment of ST and classical logic coincide at the inferential level but diverge at the metainferential level, they phrase the difficulty:

The reason for this is... that the previously discussed phenomenon can be replicated. By this we mean that-just like ST is a system which coincides with Classical Logic at the inferential level, but not at the metainferential level-it 
is possible to obtain a system which coincides with Classical Logic at the inferential and the metainferential level, but which does not coincide with it at the level of the metametainferences, i.e. of the inferences between metainferences. [3, p. 96]

A clear problem, of course, is that each stage in the construction of Barrio et al. includes a necessary point of disharmony, i.e., the prima facie classicality of metainferences of any particular tier will be revealed as nonclassical in the next tier up.

We propose that the most direct way to defuse this problem-i.e., to eliminate the eventual non-classicality that necessarily appears at some level in any of the metainferential logics described by Barrio et al.- is to define a limit in which all inferential features share a uniform foundation. This calls for a limit to the hierarchy.

There are several desiderata that we could expect of such a limit. For one, an adequate limit would have to meet the condition that for any tier of metainferences described by the limit, the metametainferences by which it is governed are likewise described and characterized by the limit. More succinctly, we would expect that a limit of metainferential logics would be capable of reflecting the metainferential principles that hold between metainferences of any level. For two, adequacy would require that the operands of the limit include the objects treated by any $\mathrm{ST}_{i}$, that is, metainferences of any finite tier should continue to be represented in the limit.

There are several a priori plausible approaches with which to define a limit. The simplest is to simply gather the individually valid metainferences of each logic in the hierarchy and take their union. Alternatively, we could propose that one takes the metainferential logic whose operands are infinitary metainferences of length $\omega$.

Both of these seem to have drawbacks. The former approach involves a sort of inertness between objects of different tiers and misses the types of mixed inferences that we have described. The latter approach would introduce infinitary objects as its operands and would suffer the same conceptual difficulties that one faces when considering what a non-standard Gödel number is meant to encode. Although (as a referee has kindly pointed out), non-standard Gödel numbers are well-defined mathematical objects, it is far from clear how the proofs they encode resemble logical reasoning as performed by humans.

\subsection{Semantics}

The metainferential logics in the ST hierarchy are concerned with representing the validity of particular metainferences. Consequently, the utility of any of these systems is in large part determined by the feasibility of determining when a metainference is valid.

Unfortunately, the impracticality of determining validity or finding counterexamples grows rapidly as one rises through the hierarchy. In [3], validity for a metainference in the logic TSST requires evaluating the validity of subobjects in both TS and ST. The pattern of alternating between strict and tolerant while climbing back down the hierarchy is repeated at each stage. 
To illustrate, suppose that we are working with a metainference in the metainferential logic Strict/Tolerant . $_{\text {. }}$

$$
\left\{\Phi_{0}, \ldots, \Phi_{n-1}\right\} \Rightarrow \Phi_{n}
$$

The metainference is valid if every Strict 3 -verifier for each $\Phi_{i}$ (with $i<n$ ) is a Tolerant 3 -verifier for $\Phi_{n}$. The sensible approach to showing validity would work from the hypothesis that a Kleene-Kripke valuation $v$ Strict $_{3}$-verifies all $\Phi_{i}$ and demonstrates that $v$ must Tolerant 3 -verify $\Phi_{n}$.

Yet the rapid alternations between strictness and tolerance (on the one hand) and verifiers and counterexamples (on the other) entails that even to fully articulate these conditions soon becomes treacherously complicated. Each $\Phi_{i}$ is itself, of course, a metainference of the form

$$
\left\{\Phi_{i, 0}, \ldots, \Phi_{i, m_{i}-1}\right\} \Rightarrow \Phi_{i, m_{i}}
$$

whence the hypothesis that $v$ is a Strict 3 -verifier for $\Phi_{i}$ decomposes into the hypothesis that $v$ is either a Tolerant 2 -counterexample for a $\Phi_{i, j}$ (where $j<m_{i}$ ) or is a Strict $_{2}$-verifier for $\Phi_{i, m_{i}}$. But these objects $\Phi_{i, j}$ themselves are metainferences whose operands are sequents. Hence, the hypothesis must be stated in terms of even finer conditions on whether $v$ is, e.g., a Strict ${ }_{1}$-verifier for some formula in the antecedent of the antecedent of $\Phi_{i, j}$.

Suffice it to say that the mere act of informally describing the steps required to establish validity of a metainference in Strict/Tolerant ${ }_{4}$ is dizzying. The actual execution of the task of demonstrating validity is nigh intractable. Given the clear and recursive definitions in [3], writing a program to search for counterexamples may be straightforward but attempting to practically leverage the calculus-proving the validity of some metainference with pen-and-paper, for example-would be unreasonable.

In practice, this intractability serves to obscure the conceptual basis of any member in the hierarchy-going through exercises is regarded as the most direct path to conceptually grasping a mathematical object. Providing a uniform account that is as tractable at level 8 as it is at level 2 is, we believe, a worthy project. Even if the philosophical merit of our preferred interpretation is found lacking, we believe that the simplicity and elegance of the semantics described below should be welcomed by proponents of the approach of Barrio and his collaborators.

\section{An Alternative Approach}

We now wish to supply an alternative to the methods described in [3] of a metainferential logic that solves the difficulties we have just described. Our approach will encompass and respect every level in the Barrio, Pailos, and Szmuc's hierarchy but will bring a uniform semantics to the logic that acknowledges mixed inferences, provides a limit to the hierarchy, and drastically simplifies the semantics.

The trick in what we will describe is simple: We will show that for all of the prima facie impracticality in the approach outlined in [3] - the complicated alternations between strict and tolerant metainferential logics of lower and lower levels-all of 
the information in these alternations is encoded in the humble three-valued material conditional of LP described in [14].

\subsection{Semantics, Generalized}

In order to establish semantics for objects in $\mathcal{L}_{\text {mix }}$, we first introduce natural generalizations of strict and tolerant counterexamples and verifiers.

Definition 17 For a sequent $\Psi, v$ is a Tolerant ${ }^{\star}$-counterexample (respectively, Tolerant ${ }^{\star}$-verifier, Strict ${ }^{\star}$-counterexample, Strict ${ }^{\star}$-verifier) when $v$ is a Tolerant ${ }_{1-}$ counterexample (respectively, Tolerant ${ }_{1}$-verifier, Strict ${ }_{1}$-counterexample, Strict ${ }_{1}$ verifier). For any other $\Psi=\left\{\Phi_{0}, \ldots, \Phi_{i}\right\} \Rightarrow \Phi, v$ is a:

Tolerant $^{\star}$-counterexample to $\Psi$ if $v$ is a Strict ${ }^{\star}$-verifier for each $\Phi_{i}$ and a Tolerant ${ }^{\star}$ counterexample to $\Phi$.

Strict ${ }^{\star}$-counterexample to $\Psi$ if $v$ is a Tolerant ${ }^{\star}$-verifier for each $\Phi_{i}$ and a Strict ${ }^{\star}$ counterexample to $\Phi$.

This enables us to define a metainferential logic that extends the ST intuitions to all levels of metainferential reasoning, including mixed reasoning.

Definition 18 We define $\mathrm{ST}_{\omega}$-validity of a $\Psi \in \mathcal{L}_{\text {mix }}$ for sequents and metainferences in parallel.

- For $\Psi=[\Gamma-\Delta], \Psi$ is $\mathrm{ST}_{\omega}$-valid when every $v$ that Strict ${ }^{\star}$-verifies all formulae in $\Gamma$ is a Tolerant ${ }^{\star}$-verifier for some formula in $\Delta$.

- For $\Psi=\left\{\Phi_{0}, \ldots, \Phi_{i}\right\} \Rightarrow \Phi, \Psi$ is $\mathrm{ST}_{\omega}$-valid when every $v$ that Strict ${ }^{\star}$-verifies all $\Phi_{j}$ is a Tolerant ${ }^{\star}$-verifier for $\Phi$.

The adequacy should be easily seen by noting that the general verifiers and counterexamples in Definition 17 agree with the earlier verifiers and counterexamples tailored to any particular tier in appropriate cases.

Thus, we are immediately guaranteed agreement with the systems in the ST hierarchy:

Theorem 1 For a non-mixed metainference $\Psi \in \mathcal{L}_{i}$,

$$
\Psi \text { is } \text { Strict }_{i} / \text { Tolerant }_{i} \text {-valid iff } \Psi \text { is } \mathrm{ST}_{\omega} \text {-valid }
$$

One may reasonably ask why we consider $\omega$ to be an adequate ordinal for the task of defining a limit to the hierarchy. Surely, one could define hierarchies with greater ordinals. While this indeed worth considering - and we look forward to returning to this question-we find $\mathrm{ST}_{\omega}$ to satisfy a goldilocks principle. For one, $\omega$ is sufficiently large in the sense that for any finite set of metainferences, any metainference asserting that a conclusion follows therefrom is expressible in its language. On the other hand, moving past $\omega$ to a greater ordinal $\beta$ would require that $\mathrm{ST}_{\beta}$ includes some infinitary metainferences. As we have indicated at several points, it is not obvious 
that a reasoner with finite resources can survey—-much less make use of - a proof of infinite length.

\subsection{The Subtlety of the LP Conditional}

Two things can be said of the system we have defined in terms of Strict ${ }^{\star}$ and Tolerant ${ }^{\star}$ verifiers and counterexamples: First, it is at least adequate to the task of governing mixed inferences in a way that is harmonious with the individual strict-tolerant logics in the ST hierarchy. Second, the semantics as we have described them do nothing to solve the problem of tractability. We now turn to showing how a simple approach suffices.

Recall the three-valued truth function for the material conditional of Priest's LP of [14]:

\begin{tabular}{c|ccc}
$\supset$ & 1 & $\frac{1}{2}$ & 0 \\
\hline 1 & 1 & $\frac{1}{2}$ & 0 \\
$\frac{1}{2}$ & 1 & $\frac{1}{2}$ & $\frac{1}{2}$ \\
0 & 1 & 1 & 1
\end{tabular}

We will show that the function $f_{\mathrm{LP}}$ provides all the machinery to define a straightforward semantics for $\mathrm{ST}_{\omega}$ (and, consequently, any metainferential logic in Barrio, Pailos, and Szmuc's hierarchy).

As an intermediate step, however, our countenancing mixed inferences requires that we extend the translation of [8] or [3] to accommodate such objects as well.

Given a translation function ${ }_{-}{ }^{\tau}$, we can map metainferences to first order formulae.

Definition 19 For a sequent $[\Gamma \succ \Delta],[\Gamma \succ \Delta]^{\tau}=d f \wedge \Gamma \supset \bigvee \Delta$.

Definition 20 For any non-sequent object in $\mathcal{L}_{\text {mix }}$ of the form $\left\{\Phi_{0}, \ldots, \Phi_{i}\right\} \Rightarrow \Phi$, $\left(\left\{\Phi_{0}, \ldots, \Phi_{i}\right\} \Rightarrow \Phi\right)^{\tau}=\bigwedge_{j \leq i} \Phi_{j}^{\tau} \supset \Phi^{\tau}$

In other words, the material conditional $\supset$ is used to translate both the sequent separator and meta-sequent separators of any level.

As an auxiliary to the following proofs, we explicitly identify the following obvious features of the truth function $f_{\mathrm{LP}}$, which may be confirmed by consulting its truth-table:

Observation 1 The three-valued function $f_{\mathrm{LP}}^{\supset}$ enjoys the following properties:

- $f_{\mathrm{LP}}(x, y)=0$ iff $x=1$ and $y=0$

- $f_{\mathrm{LP}}^{\supset}(x, y) \in\left\{0, \frac{1}{2}\right\}$ iff $x \neq 0$ and $y \neq 1$

This observation provides sufficient leverage to establish a theorem, the proof of which will require that we define a notion of depth:

Definition 21 For a $\Psi \in \mathcal{L}_{\text {mix }}$, the depth of $\Psi($ depth $(\Psi))$ is defined recursively:

- $\operatorname{depth}(\Psi)=1$ for $\Psi \in \mathcal{L}_{1}$ (i.e. sequents) 
- $\operatorname{depth}\left(\left\{\Phi_{0}, \ldots, \Phi_{i}\right\} \Rightarrow \Phi\right)=\max \left(\operatorname{depth}\left(\Phi_{0}\right), \ldots, \operatorname{depth}\left(\Phi_{i}\right), \operatorname{depth}(\Phi)\right)+1$

Theorem 2 For all $\Psi \in \mathcal{L}_{\operatorname{mix}}$ and all Kleene-Kripke valuations $v$, the following facts hold:

- Fact I: $v$ is a Tolerant $^{\star}$-counterexample to $\Psi$ iff $v\left(\Psi^{\tau}\right)=0$.

- $\quad$ Fact II: $v$ is a Strict $^{\star}$-counterexample to $\Psi$ iff $v\left(\Psi^{\tau}\right) \in\left\{0, \frac{1}{2}\right\}$.

Proof We prove this by an induction on the depth of $\Psi \in \mathcal{L}_{\text {mix }}$. In case depth $(\Psi)=$ $1, \Psi$ is a sequent $[\Gamma>\Delta]$, whence the basis step requires establishing the facts for ST and TS (in which case Tolerant ${ }^{\star}$ and Strict ${ }^{\star}$ reduce to Tolerant ${ }_{1}$ and Strict ${ }_{1}$, respectively). Although the basis step isn't novel, its rehearsal should serve as a conceptual guide to the following double induction. Then we establish Facts I and II individually for sequents $\Psi$ :

Basis for Fact I: $v$ is a Tolerant 1 (i.e., ST) counterexample to sequent $\Psi$ iff $v[\Gamma]=\{1\}$ and $v[\Delta]=\{0\}$. This holds iff $v(\bigwedge \Gamma)=1$ and $v(\bigvee \Delta)=0$. But Observation 1 ensures that this holds iff $f_{\mathrm{LP}}(\bigwedge \Gamma, \bigvee \Delta)=0$, i.e., this holds iff $v\left(\Psi^{\tau}\right)=0$.

Basis for Fact II: $v$ is, on the other hand, a Strict ${ }_{1}$ (i.e., TS) counterexample to $\Psi$ iff $v[\Gamma] \subseteq\left\{\frac{1}{2}, 1\right\}$ and $1 \notin v[\Delta]$. These conditions are jointly equivalent to the case in which $v(\bigwedge \Gamma) \neq 0$ and $v(\bigvee \Delta) \neq 1$. By Observation 1 , such cases are exactly those in which $f_{\mathrm{LP}}(v(\bigwedge \Gamma), v(\bigvee \Delta)) \in\left\{0, \frac{1}{2}\right\}$, i.e., when $v\left(\Psi^{\tau}\right) \in\left\{0, \frac{1}{2}\right\}$.

Now, we establish that Facts I and II are true for all $n$, assuming as induction hypothesis that the properties hold for all $m$ less than $n$. In such cases, $\Psi$ will be of a metainference of the form $\left\{\Phi_{0}, \ldots, \Phi_{j}\right\} \Rightarrow S$. Again, we treat the two cases individually:

Induction Step for Fact I: By definition, $v$ is a Tolerant ${ }^{\star}$-counterexample to $\Psi$ iff $v$ is a Strict ${ }^{\star}$-verifier for each $\Phi_{i}$ and $v$ is a Tolerant ${ }^{\star}$-counterexample to $\Phi$. By the induction hypothesis, the former conjunct holds iff for each $\Phi_{i}, v\left(\Phi_{i}^{\tau}\right)=1$ (which, in turn, holds iff $\left.v\left(\bigwedge_{i} \Phi_{i}^{\tau}\right)=1\right)$. Similarly, the induction hypothesis ensures the equivalence between the second conjunct the case in which $v\left(\Phi^{\tau}\right)=0$. Hence, the previous conjunction is equivalent to the case in which both $v\left(\bigwedge_{i} \Phi_{i}^{\tau}\right)=1$ and $v\left(\Phi^{\tau}\right)=0$. But by Observation 1, these conditions hold iff $f_{\mathrm{LP}}^{\supset}\left(v\left(\bigwedge_{i} \Phi_{i}^{\tau}\right), v\left(\Phi^{\tau}\right)=\right.$ 0 , i.e., precisely when $v\left(\Psi^{\tau}\right)=0$.

Induction Step for Fact II: Again, $v$ is a Strict ${ }^{\star}$-counterexample to $\Psi$ precisely when $v$ is both a Tolerant ${ }^{\star}$-verifier for every $\Phi_{i}$ as well as a Strict ${ }^{\star}$-counterexample for $\Phi$. By the induction hypothesis, the former conjunct is holds iff for no $\Phi_{i}$ is $v\left(\Phi_{i}^{\tau}\right)=0$; similarly, the latter conjunct holds iff $v\left(\Phi^{\tau}\right) \in\left\{0, \frac{1}{2}\right\}$. Furthermore, these two cases are equivalent to the cases in which $v\left(\bigwedge_{i} \Phi_{i}^{\tau}\right) \neq 0$ and $v\left(\Phi^{\tau}\right) \neq 1$, respectively. Again, Observation 1 shows this conjunction to be equivalent to the case in which $f_{\mathrm{LP}}\left(v\left(\bigwedge_{i} \Phi_{i}^{\tau}\right), v\left(\Phi^{\tau}\right)\right) \in\left\{0, \frac{1}{2}\right\}$, i.e., when $v\left(\Psi^{\tau}\right) \in\left\{0, \frac{1}{2}\right\}$. 
From this, we are able to establish what is at once a correspondence theorem and an alternative semantics for $\mathrm{ST}_{\omega}$.

First, let us recall the logic LPTT_-Priest's LP with a transparent truth predicatedescribed semantically in $[7$, p. 846$]$ so that:

Definition 22 For sets of first-order formulae of $\mathcal{L}_{\text {base }} \Gamma, \Delta, \Gamma \vDash_{\text {LPTT }}$ if for all Kleene-Kripke valuations $v$, whenever $v[\Gamma] \subseteq\left\{\frac{1}{2}, 1\right\}$, then for some $\varphi \in \Delta, v(\varphi) \in$ $\left\{\frac{1}{2}, 1\right\}$.

Importantly, when no formulae in $\Gamma$ or $\Delta$ include an instance of the truth predicate, LPTT coincides with LP.

Theorem 3 For all metainferences $\Psi \in \mathcal{L}_{\mathrm{mix}}$, the following are equivalent:

- $\Psi$ is $\mathrm{ST}_{\omega}$-valid

- $\quad \vDash_{\text {LPTT }} \Psi^{\tau}$

Proof Without loss of generality, let $\Psi$ be of the form $\left\{\Phi_{0}, \ldots, \Phi_{j}\right\} \Rightarrow \Phi$. $\Psi$ will be $\mathrm{ST}_{\omega}$-valid precisely when for all Strict ${ }^{\star}$-verifiers $v$ of each $\Phi_{i}, v$ is also a Tolerant ${ }^{\star}$ verifier for $\Phi$. By Theorem 2 and the properties of conjunction, this is equivalent to the case in which for all $v$ such that $v\left(\bigwedge_{i} \Phi_{i}^{\tau}\right)=1$, also $v\left(\Phi^{\tau}\right) \neq 0$. But, as made explicit in Observation 1, this holds iff for no $v, f_{\mathrm{LP}}\left(v\left(\bigwedge_{i} \Phi_{i}^{\tau}\right), v\left(\Phi^{\tau}\right)=0\right.$, i.e., $\Psi^{\tau}$ can never receive an assignment to 0 . But this is just to say that $\Psi^{\tau}$ is valid in (and hence a theorem of) LPTT.

As $\mathrm{ST}_{\omega}$ includes each strict-tolerant metainferential logic in the hierarchy, we can establish that this semantics applies to the individual metainferential logics.

Corollary 1 For all formulae $\Psi$ from $\mathcal{L}_{n}$ (i.e., a sequent from $\mathcal{L}_{1}$ or a metainference) and all Kleene-Kripke valuations $v$, the following facts hold:

- $\quad$ Fact I: $v$ is a Tolerant $_{n}$-counterexample to $\Psi$ iff $v\left(\Psi^{\tau}\right)=0$.

- Fact II: $v$ is a Strict $_{n}$-counterexample to $\Psi$ iff $v\left(\Psi^{\tau}\right) \in\left\{0, \frac{1}{2}\right\}$.

Corollary 2 For all formulae $\Psi$ from $\mathcal{L}_{n}$, the following are equivalent:

- $\Psi$ is Strict/Tolerant $_{n}$-valid

- $\quad \vDash_{\text {LPTT }} \Psi^{\tau}$

There are many potential lenses from which one may view the consequences of the foregoing observations.

It is, for example, surprising that the complicated nature of these metainferential consequence relations should be reduced to something so simple, i.e., that there exists an immediate translation between the elaborate and recursively-defined consequence

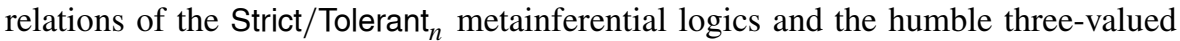
truth function $f_{\mathrm{LP}}^{\supset}$. One might be inclined to take a dismissive stance concerning 
the ST hierarchy and infer that, for all its apparent depth, it is "merely" a dressedup version of the pedestrian LP. Despite the apparent sophistication of the notions of strict and tolerant verifiers and counterexamples of increasing scale, one might conclude, such sophistication only obscured its simple origins.

We favor the opposite approach. The core concepts upon which the ST hierarchy rests need not be considered tarnished by this correspondence; indeed, they are clearly meaningful and successfully lift the core ideas of ST to a unified account of metainference at all levels. What is astonishing is not that such an intriguing milieu should be so easily reduced to LP, but rather, that such a prima facie uncomplicated logic like LP should so efficiently encode these robust concepts into the disarmingly unpretentious material conditional $f_{\mathrm{LP}}$.

\subsection{Dissolving the Limitations}

In the foregoing section we had described three formal limitations of Barrio, Pailos, and Szmuc's hierarchies and set out to show that our suggested approach served to improve on these authors' core idea.

First, we had claimed that the lack of interaction between any two distinct tiers of metainferences among the hierarchy was a limitation in the sense that the regimentation is artificial and fails to account for common metainferential patterns. Insofar as the operands of the metainferential logic we have defined include mixed inferences, we believe that our proposal is a better fit to the patterns of reasoning actually employed in practice.

Second, we had claimed that the ST hierarchy lacked a natural limit; one could propose e.g. that one takes the union of all metainferential logics in the hierarchy, but with no interaction between the tiers, desired dynamism would be absent. Our proposal does provide a natural and reasonable limit; it agrees with the valid metainferences of each tier while appropriately extending the same intuitions to metainferences that make use of inferences of different levels.

Finally, we had described a problem for the utility of any of the members of the hierarchy, i.e., that the unwieldiness in the recursive definitions of verifiers and falsifiers made their use impractical in e.g. finding counterexamples or semantically proving validity. The uniform semantics we have described require no more overhead than any three-valued logic, eliminating the problem of utility. Moreover, this unwieldiness obscures the uniformity that our semantics wears on its face, winning our account additional elegance and clarity.

Along these lines alone, we feel that the merit of the foregoing results should be clear. Nevertheless, our interest in our proposal runs deeper than merely its utility as a formalism; we also believe that it clears new ground for a satisfying fulfillment of the strict-tolerant intuitions, to which we will now turn.

\section{Deep ST}

We believe that the foregoing has more weight than the mere resolution of a handful of difficulties with the original ST hierarchy. Indeed, we believe that it sets the stage 
on which the strict-tolerant intuitions can be expanded and promoted to an authentic theory of reasoning.

Consider two activities: Defining a formal calculus and describing a philosophical theory of inference. Although these activities may be closely related-often the suitability of a formal calculus is judged on the degree to which it corresponds to a theory of reasoning - there are distinctions between them. One crucial component of the distinction, we think, is the whether there is an expectation of constancy. Although the rules one defines for some novel sequent calculus may be more-or-less plausible, their scope is restricted to the manipulation of symbols; one is not charged with inconstancy for deviating from these rules when describing its metatheory. On the other hand, justifying any particular theory of inference requires that one reason about its content and consequences, and this reasoning necessarily presupposes some facts about inference.

To provide an example, consider two ways of interpreting intuitionistic logic. One may be interested in a intuitionistic logic qua calculus with one's interest limited to finding its model theory mathematically interesting. In such a case, one can hardly be called unprincipled for appealing to excluded middle during a proof of some feature of its Kripke models. But if one is a proponent of constructivism as a theory of reasoning, constancy demands that one avoid violating intuitionistic principles at any stage of reasoning.

Thus, just as a constructive mathematician takes intuitionistic logic to be governed by an intuitionistic metatheory, if the strict-tolerant theorist accepts that metainferences themselves (and metametainferences, and so on) are governed by strict-tolerant conceptions of consequence, then ST can be viewed as classical all the way down.

With a limit-i.e., a uniform semantics that governs all tiers of inference and meta-inference-in place, the ST-theorist is free to say that the bounds consequence principles governing sequents in ST are not logical artifacts or formal curiosities. Rather, the dedicated ST-theorist can say that these intuitions are intuitions about reasoning. We will call the position induced by this picture deep ST.

\subsection{Constancy in Inferential Principles}

If one intends for one's formal calculus to authentically reflect a true theory of inference, a constancy with respect to inferential principles seems imperative. The canonical example is, of course, intuitionism. Although such constancy is clear in Brouwer's formulation-which was not reliant on a symbolic calculus but rather described a holistic theory of practice - a constancy between the formalism and its study is clear from his inheritors as well. ${ }^{8}$ E.g., Heyting's archetypal intuitionist describes the relationship between constructivism and its formalization like so:

[I]ntuitionism proceeds independently of the formalization, which can but follow after the mathematical construction. [12, p. 5]

\footnotetext{
${ }^{8}$ For discussion of the relationship between reasoning and mathematical practice in Brouwer, see [4], reprinted with commentary in [5].
} 
In other words, although the intuitionistic calculus may be designed to represent intuitionistic practice, it remains a mathematical object and must be studied along the intuitionistic principles it reflects.

We propose that the uniformity of the above semantic analysis of the limit $\mathrm{ST}_{\omega}$ reveals the logic to enjoy this very type of constancy. In the same way that Heyting's intuitionist of [12] insists on general constructive principles both in the formal calculus and reasoning about the calculus, so does $\mathrm{ST}_{\omega}$ supply the apparatus for the committed ST-theorist to lift the strict-tolerant approach from the sequent calculus to any tier of reasoning. $\mathrm{ST}_{\omega}$ thus arguably clears a space for the strict-tolerant intuition to evolve from a position about formal logic to a position about reasoning.

When we turn our attention to bounds consequence as the core of the stricttolerant intuition, this constancy enjoys especially pleasing consequences. As we have suggested, the standard reading of ST gives a bounds consequence reading to the deducibility of a particular sequent but retains an operational reading to metainferences.

Given the uniform semantics for every tier of metainference, we are licensed to lift the bounds consequence interpretation from sequents to metainferences and provide a bounds consequence reading to all types of metainferences. (Recall that Section 4.1.1 described the shape of a bounds consequence reading to metainferences). This option frees bounds consequence from being associated merely with metainferences of a particular level, allowing its full realization as a theory concerning consequence without restriction.

This may give one pause-we have elsewhere suggested that a consequence is that deep ST is the total materialization of reasoning - but this material flavor is part and parcel of the notion of bounds. If one feels free to interpret the sequent-an object already loaded with an inferential character-as a material position, to consider inference and metainference as sharing a common basis presupposes such a material reading of metainferences as well. In short, the approach we have described is a full-throated application of the plausible and attractive approach outlined by bounds consequence, taking the underlying thesis to its natural conclusion.

\subsection{Treatment of Paradox Outside Object Language}

As presented, the objects of metainferences of e.g., TSST are more restricted in expressivity than the first-order formulae that compose a position. While the comma separating objects in antecedent positions serves a cognate function to conjunction, notions like disjunction or negation are lacking. Such weakened expressivity is thus inherited by our presentation of $\mathrm{ST}_{\omega}$.

In practice, however, it makes sense to consider such operations when considering metainferences. The following seem consonant with practice:

- $\quad$ Either the metainference $\Phi$ is valid or the metainference $\Psi$ is valid

- The metainference $\Xi$ is not valid

So a very natural project to embark on-whether or not one accepts the approach we've outlined above-is to attempt to capture these very natural speech acts in the formal language itself and to study its logic. 
We will pause to say that the uniformity offered by the simple semantics for $\mathrm{ST}_{\omega}$ provides an obvious path forward. By saying that conjunction in the object language is semantically uniform with conjunction in the metalanguage, a promising semantics for $e$.g. disjunctions of metainferences is immediately available. Thus, the obvious enrichment of the language - i.e., an enrichment including objects like $\sim[\Gamma>\Delta]-$ has a corresponding semantic analysis waiting in the wings.

Although we leave such tasks for future investigation, this consideration does expose an attractive feature available to the deep ST-theorist not available to out-of-the-box ST.

Recall that the bounds-consequence reading of the derivability of a position $[\Gamma>$ $\Delta]$ is understood as the statement "it is out-of-bounds to take the position $[\Gamma>\Delta]$." This natural-language statement, of course, is truth-evaluable; it is entirely meaningful to debate whether or not a particular position is out-of-bounds. Formally, the sentence

- "It is true that: The position $[\Gamma>\Delta]$ is out-of-bounds."

would reasonably correspond to a formula of the form $T(\ulcorner\{\} \Rightarrow\{[\Gamma>\Delta]\}\urcorner)$, where $\ulcorner\{\} \Rightarrow\{[\Gamma>\Delta]\}\urcorner$ is a term denoting the derivability of the position. As the expressivity of the language is enhanced, then, it is appropriate not only to enrich the complexity of available formulae but also widen the breadth of terms.

Now, if this is a reasonable next step-and we think it is at least as reasonable than including a term naming each formula - one might expect that the open formula $\sim T(x)$ will have a fixed point among the terms that name metainferences. ${ }^{9}$ Intuitively, it is easy to come up with corresponding cases for what paradoxical applications of the truth predicate to positions would look like.

What is important here is that the approach that we have described allows us to not only extend the strict-tolerant intuition to account for mixed inferences, but also makes available new increases in expressivity. ST provides transparent truth only if we consider truth predicates applied to the object language. The deep ST-theorist will insist that logic considers our natural uses of the concept of truth in discourses in the metalanguage as well; for the deep ST-theorist, an approach such as the one described here will be necessary.

\subsection{Classicality Recovered?}

A deficiency in strict-tolerant approaches identified by Barrio and his collaborators in [3] is the ineliminability of non-classicality, that is, that the failures of Cut at some metainferential level cannot be wholly eliminated. That the appearance of nonclassicality is always guaranteed at some level for any of their metainferential logics guarantees the general approach has little chance of being thought to be classical.

The results of this paper, we submit, provide a serviceable remedy to this concern. For every tier of metareasoning covered in a logic among the hierarchy, the semantics encompasses not only that tier but also the metareasoning about that tier.

\footnotetext{
${ }^{9}$ Certainly, adding such a predicate to any $\mathrm{ST}_{i}$ in the hierarchy would yield a fixed point. We do not know that one has a guarantee that there will be such fixed points and leave this only as conjecture.
} 
As a corollary to Theorem 3 , we have the following result:

Corollary 3 For all metainferences $\Psi \in \mathcal{L}_{\text {mix }}$ including no instances of the truth predicate, the following are equivalent:

- $\Psi$ is $\mathrm{ST}_{\omega}$-valid

- $\vdash_{\mathrm{CL}} \Psi^{\tau}$

Proof If $\Psi$ includes no instances of the truth predicate, then $\Psi^{\tau}$ is formulated in a standard first-order language. Definition 22 thus entails that $\Psi^{\tau}$ is LPTT-valid precisely when it is LP-valid, and thus a theorem of LP. It is well-known (since [14] and discussed in this context in [16]) that LP and CL share the same theorems. Thus, from this equivalence and Corollary 2, this corollary follows.

From Corollary 3, we see that for any metainference governing strictly classical formulae, the metainferential behavior is indeed entirely classical. We may observe, e.g., not only that Cut is valid, but also that the metainferential counterpart for any level of inference is also valid.

It seems to be an entirely rational position to take that if one is to reject the classicality of $\mathrm{ST}_{\omega}$ - or to assert that its introduction of a truth operator abrogates the classicality of its foundation-one must be able to provide an example of how the two differ. And, given the above result, such a case cannot be provided.

If one objects to the deep ST-theorist's refusal to infer the validity of $[>-$ from the validity of $[\lambda \succ]$ and $[\succ \lambda]$, the deep ST-theorist can readily reply that, since they endorse the strict-tolerant standard of reasoning at all levels, it follows that their endorsement of ST at the level of metametainferences licenses their simultaneous acceptance of the validity of $[>$ ] from the validity of $[\lambda>\quad]$ and $[-\lambda]$. Moreover, the deep ST-theorist is always prepared to falsify the claim that there is a level $n$ such that there is a truly non-classical instance of reasoning which takes place at that level.

Briefly, Corollary 3 ensures that we can never really provide a concrete instance of metareasoning that is inherently non-classical in the strictly classical language. Furthermore, notice that the deep ST-theorist can argue that their logic is just classical logic to the same extent that the standard ST-theorist is able to and then some. If we take a logic to be a collection of valid inferences, then both theorists are entitled to the same conclusion. The advantage that the deep ST-theorist holds over the standard ST-theorist is that while the latter is not in a position to argue that they can overturn the criterion for the identity of logics proposed in [3], the former definitely can. There is a sense in which the deep ST-theorist can argue that their preferred theory of reasoning is classical at all levels.

Because it is not obvious that one can stop the deep ST-theorist from applying Corollary 3 at any given point of the hierarchy, one cannot argue that their reasoning at that level is inherently non-classical. After all, if the classical theorist is to charge the deep ST-theorist with non-classicality, she should point out a classical thesis that the 
deep ST-theorist does not accept. But insofar as Corollary 3 demonstrates a type of conservativity of $\mathrm{ST}_{\omega}$ over classical reasoning, such a counterexample does not exist.

Another possible way of resisting the deep ST-theorist might involve pointing out that their reasoning being classical at all levels is a technicality, rather than a feature "naturally" built into the theory. This, we believe, would amount to a departure from the original criterion that appears in [3]. Although this might trigger a necessary, perhaps expected move towards more sophisticated identity criteria, it would still be a rejection of the intuitive requirement that it suffices for two logics to match in their validities at all levels to be identical. ${ }^{10}$

Finally, we would like to point out that it is Dicher and Paoli's criterion which poses a challenge for the deep ST-theorist. Since their position is one which prioritizes abstract consequence relations over collections of (meta)inferences, it might come across as immune to the results stated in Corollary 3. The immediate reaction, from the point of view of Dicher and Paoli's position, is that the deep ST-theorist has simply chosen to endorse LP as their preferred abstract logical consequence relation, regardless of the kinds of objects of reasoning they might be dealing with. In that case, deep ST-theorists might as well be renamed deep LP-theorists, opposed to standard LP-theorists, who endorse LP as their preferred consequence relation exclusively at the level of formulas.

\section{Conclusions}

We have intended the foregoing to serve several purposes and believe that we have made a compelling case for taking the approach outlined above seriously.

From a formal perspective, the results provide some improvements to-and a natural extension complementing - the approach outlined in [3]. The application of the LP conditional as an interpretation for sequent separators and meta-sequent separators should provide a simplification for working with any of these logics and, we hope, may serve as a uniform and feasible lens clarifying Barrio, Pailos, and Szmuc's hierarchy.

From a philosophical perspective, the results clear a space in which the stricttolerant intuition can be extended to a robust philosophical position concerning reasoning in general. The deep ST theorist, like the intuitionist, has the semantic tools to illustrate how bounds consequence applies to all reasoning and that the class of paradoxes solved by the strict-tolerant approach do not stop at the object language, but address those induced by truth predicates in the metalanguage as well.

There is clearly more work to do, of course. We have indicated a wish to e.g. extend the formalization of the metalanguage to enhance the expressivity. It makes sense to say that if a sequent is valid then either of two other sequents must be valid, something not expressible in the metalanguage of [3]. We suspect that results like Theorem 3 will immediately carry over to extend to these cases as well.

\footnotetext{
${ }^{10} \mathrm{~A}$ detailed overview of this statement is unfortunately outside of the bounds of this paper.
} 
Open Access This article is licensed under a Creative Commons Attribution 4.0 International License, which permits use, sharing, adaptation, distribution and reproduction in any medium or format, as long as you give appropriate credit to the original author(s) and the source, provide a link to the Creative Commons licence, and indicate if changes were made. The images or other third party material in this article are included in the article's Creative Commons licence, unless indicated otherwise in a credit line to the material. If material is not included in the article's Creative Commons licence and your intended use is not permitted by statutory regulation or exceeds the permitted use, you will need to obtain permission directly from the copyright holder. To view a copy of this licence, visit http://creativecommons.org/licenses/by/4.0/.

\section{References}

1. Avron, A. (1988). The semantics and proof theory of linear logic. Theoretical Computer Science, 57(2-3), 161-184.

2. Barrio, E., Rosenblatt, L., \& Tajer, D. (2015). The logics of strict-tolerant logic. Journal of Philosophical Logic, 44(5), 551-571.

3. Barrio, E., Pailos, F., \& Szmuc, D. (2020). A hierarchy of classical and paraconsistent logics. Journal of Philosophical Logic, 49(1), 93-120.

4. Brouwer, L. E. J. (1928). Mathematik, Wissenschaft und Sprache. Monatshefte für Mathematik und Physik, 36, 153-164.

5. Brouwer, L. E. J. (1996). Mathematics, science, and language. In W. Ewald (Ed.) From Kant to Hilbert, (Vol. II pp. 1170-1185). Oxford: Oxford University Press.

6. Cobreros, P., Egré, P., Ripley, D.., \& van Rooij, R. (2012). Tolerant, classical, strict. Journal of Philosophical Logic, 41(2), 347-385.

7. Cobreros, P., Egré, P., Ripley, D.., \& van Rooij, R. (2014). Reaching transparent truth. Mind, 122(488), 841-866.

8. Dicher, B., \& Paoli, F. (2018). ST, LP, and tolerant metainferences. In T. M. Ferguson, \& C. Baskent (Eds.) Graham Priest on Dialetheism and Paraconsistency (pp. 383-407). Cham: Springer.

9. Dummett, M. (1978). Truth and other enigmas. London: Gerald Duckworth \& Company.

10. Fjellstad, A. (2016). Naive modus ponens and failure of transitivity. Journal of Philosophical Logic, 45(1), 65-72.

11. French, R. (2016). Structural reflexivity and the paradoxes of self-reference. Ergo, 3(5), 113-131.

12. Heyting, A. (1976). Intuitionism: An Introduction, 3rd edn. Amsterdam: North-Holland Publishing.

13. Mares, E., \& Paoli, F. (2014). Logical consequence and the paradoxes. Journal of Philosophical Logic, 43(2-3), 439-469.

14. Priest, G. (1979). The logic of paradox. Journal of Philosophical Logic, 8(1), 219-241.

15. Restall, G. (2005). Multiple conclusions. In P. Hajek, L. Valdes-Villanueva, \& D. Westerståhl (Eds.) Logic, Methodology, and Philosophy of Science: Proceedings of the Twelfth International Congress (pp. 189-205). London: Kings College.

16. Ripley, D. (2012). Conservatively extending classical logic with transparent truth. Review of Symbolic Logic, 5(2), 354-378.

17. Ripley, D. (2013). Paradoxes and failures of cut. Australasian Journal of Philosophy, 91(1), 139-164.

18. Routley, R. (1982). Relevant Logics and their Rivals Vol. 1. CA: Ridgeview Publishing, Atascadero.

19. Zardini, E. (2013). Naive modus ponens. Journal of Philosophical Logic, 42(4), 575-593.

Publisher's Note Springer Nature remains neutral with regard to jurisdictional claims in published maps and institutional affiliations. 